\title{
Una mirada comunitaria en la escuela: el diagnóstico del barrio como motor de arranque para proyectos de Aprendizaje-Servicio
}

\section{A community look at the school: the diagnosis of the neighbourhood as a starter for Service-Learning projects}

\author{
Cristina Arriaga-Sanz, Alberto Cabedo-Mas, ${ }^{* *}$ Óscar Chiva-Bartoll, ${ }^{* * *}$ Lidón Moliner-Miravet $^{* * * *}$
}

Recibido: 25 de octubre de 2020 Aceptado: 15 de enero de 2021 Publicado: 27 de julio de 2021

To cite this article: Arriaga-Sanz, C.; Cabedo-Mas, A.; Chiva-Bartoll, O. y Moliner-Miravet, L. (2021). Una mirada comunitaria en la escuela: el diagnóstico del barrio como motor de arranque para proyectos de Aprendizaje-Servicio. Márgenes, Revista de Educación de la Universidad de Málaga. 2 (2), 100-115

DOI: http://dx.doi.org/10.24310/mgnmar.v2i2.10574

\section{RESUMEN}

El Aprendizaje-Servicio (ApS) constituye una experiencia apropiada para consolidar una mirada sociocomunitaria de la educación, ya que propicia la participación activa, reflexiva y crítica de todas las personas implicadas. Este artículo muestra la fase de diagnóstico de un barrio donde se aplica un programa de Aps, y las aportaciones que suponen en un programa concreto. A nivel metodológico, se justifica y argumenta la necesidad de establecer un diagnóstico del contexto, describiendo enfoque, proceso y criterios empleados. Como resultado, se describen los instrumentos que facilitan la recogida de información de cada colectivos. En conclusión, el artículo hace evidente la necesidad de implementar, siempre que se aspire a aplicar proyectos de ApS de calidad, una fase de diagnóstico bien diseñada y ajustada a las circunstancias de cada contexto. El ApS constituye una experiencia idónea para una mirada comunitaria, ya que propicia la participación activa, reflexiva y crítica de todas las personas implicadas, pero una propuesta trasformadora que implique la actuación de las familias y la comunidad tiene que ir precedida por un diagnóstico del contexto donde se desenvuelve, un análisis que haga posible dinamizar este trabajo conjunto. Será necesario diseñar instrumentos que faciliten la recogida de información de los distintos colectivos, para identificar los puntos fuertes y débiles e implementar proyectos de calidad, adecuados a las circunstancias del contexto. Este es el objetivo principal de este trabajo, aportar los instrumentos que facilitan un diagnóstico del barrio donde se aplica un programa de Aprendizaje-Servicio y las aportaciones que suponen en un programa concreto.

Palabras clave: diagnóstico; aprendizaje-servicio; método pedagógico; educación comunitaria; análisis del contexto

Agradecimientos: Este estudio se ha realizado dentro del proyecto de investigación EDU2017-86311-P, subvencionado por el Ministerio de Ciencia e Innovación de España.

*Cristina Arriaga-Sanz

0000-0003-0981-2905

Euskal Herriko Unibertsitatea (España)

cristina.arriaga@ehu.eus
** Alberto Cabedo-Mas 0000-0002-3703-3848

Universitat Jaume I (España) cabedoa@uji.es
*** Óscar Chiva-Bartoll 0000-0001-7128-3560

Universitat Jaume I (España)

ochiva@uji.es
${ }^{* * * *}$ Lidón Moliner-Miravet 0000-0003-0143-898X

Universitat Jaume I (España) mmoliner@uji.es 


\section{ABSTRACT}

Service-Learning (SL) is an appropriate experience to consolidate a socio-community view of education, since it encourages the active, reflective and critical participation of all those agents involved. This article shows the diagnostic phase of a neighbourhood where a SL program is applied. At the methodological level, the need to establish a diagnosis of the context where the intervention takes place is theoretically justified and argued, describing the approach, process and criteria used for the selection of the instruments. As a result, a series of instruments are described that facilitate the collection of information from the different groups. In conclusion, the article evidences the need to implement, whenever aspiring to apply quality SL projects, a well-designed diagnostic phase adjusted to the circumstances of each context. It is clear that a transforming proposal that involves the actions of families and the community, must be preceded by a diagnosis of the context in which it takes place, it is to say, an analysis that makes it possible to energize this joint work. Therefore, it will be necessary to design instruments that facilitate the collection of information from the different groups, to identify the strengths and weaknesses, in order to implement quality projects, appropriate to the circumstances of the context. This is the main objective of this work, to provide the instruments that facilitate a diagnosis of the neighbourhood where a Service-Learning program is applied and the contributions that they imply in a specific program.

Keywords: diagnosis; service-learning; pedagogical method; community education; context analysis

\section{INTRODUCCIÓN}

La propuesta de diagnóstico de contexto investigación que ahora se presenta forma parte de un proyecto más amplio, cuyo objetivo principal es implementar un programa en una escuela de educación infantil y primaria que parte de la expresión musical, plástica y corporal como motor para la convivencia positiva y el entendimiento cultural, fomentando la conexión con la comunidad escolar y la realidad que envuelve el centro a partir de una propuesta de Aprendizaje-Servicio (ApS). Así, se trabaja con estudiantes de educación primaria con un hilo conductor común basado en el desarrollo de proyectos de ApS. En la actualidad, aunque existen multitud de definiciones y conceptualizaciones sobre el ApS, una de las más aceptadas y extendidas sigue siendo la de Bringle y Hatcher (1995):

El Aprendizaje-Servicio es una experiencia educativa curricular en la quelos estudiantes: (a) participan en un servicio organizado que cumple con unas necesidades identificadas por la comunidad y (b) reflexionan sobre el servicio de tal manera que se promueve un conocimiento más amplio de la materia y un mayor sentido de responsabilidad cívica (p. 112).

En particular, el proyecto tiene su base filosófica en dos perspectivas educativas estrechamente vinculadas, la inclusión y la interculturalidad. La Educación Intercultural Inclusiva se concibe como un modelo educativo, tanto formal como no formal, que propicia el enriquecimiento cultural de la ciudadanía, partiendo del reconocimiento y respeto a la diversidad, a través del intercambio y el diálogo, en la participación activa y crítica para el desarrollo de una sociedad democrática basada en la equidad y la solidaridad (Sales y García, 1997).

En coherencia con esta idea, se apuesta por la vía metodológica del ApS, que amplía las áreas de actuación más allá del espacio escolar condicionado por el diseño del currículum y desarrollando acciones socioeducativas de naturaleza multidimensional que dinamicen la apertura del centro escolar a la sociedad. De esta manera, el programa de ApS que aquí nos ocupa trata de reforzar la importancia de la gestión del cambio a través de la participación local, ya que los 
procesos de mejora escolar están conectados con la comunidad. Igualmente, implica la participación de todos los interesados, los profesionales de la educación, el alumnado, las familias y los miembros de la comunidad social, para evitar la separación del aprendizaje escolar de la vida.

Una vez evidenciada la necesidad de implicar a todas las personas interesadas para fomentar su participación en proyectos de esta índole, se ha constatado la necesidad de realizar un diagnóstico del contexto, a fin de enfocar bien el proyecto a desempeñar, así como la posterior evaluación de su impacto social (Chiva-Bartoll, Santos-Pastor, Martínez-Muñoz, \& SalvadorGarcía, 2019). El contexto familiar y comunitario no puede ser obviado en el análisis de la realidad educativa (Ceballos, 2006), menos si cabe cuando establecemos propuestas de proyectos comunitarios en los centros.

La finalidad de este artículo es recoger la primera fase de este estudio, es decir, dar luz a los procesos a través de los cuáles se ha realizado un análisis del contexto donde se ubica el centro escolar. Este análisis se ha llevado a cabo a través del diseño de unos instrumentos para la recogida de información que posibilitan el diagnóstico del contexto donde se ubica el centro escolar, subrayando la importancia de llevar a cabo este diagnóstico para elaborar propuestas que dinamicen la relación entre la escuela y el barrio.

\section{2. ¿CÓMO ES LA COMUNIDAD EN LA ACTUALIDAD? ALGUNAS CARACTERÍSTICAS Y SUS IMPLICACIONES DOCENTES}

Las transformaciones sociales y los movimientos migratorios que están sucediendo en las últimas décadas sitúan el modelo de escuela actual en un escenario que incluye nuevos colectivos. Inmigrantes, diferentes estructuras familiares o minorías étnicas caracterizan el proceso de globalización y plantean una nueva realidad que supone nuevos retos educativos (Caballo y Gradaille, 2008). Estos retos conllevan necesariamente nuevas estrategias docentes y acciones pedagógicas que abran las puertas de la escuela a la sociedad desde una perspectiva holística e integradora. Hacen necesaria una mirada diferente que abarque realidades diversas y se desvincule de los patrones curriculares, tiempos y espacios de la escuela tradicional, incorporando nuevos agentes que incluyan no sólo a las familias sino también a la comunidad local, instituciones y actores que ofrecen y buscan espacios y recursos, asumiendo un rol más activo en las dinámicas educativas, enlazando las actividades escolares con las comunitarias y sociales, utilizando y rentabilizando desde la escuela los instrumentos y redes socioculturales presentes en la comunidad. En este sentido, Caballo y Gradaílle (2008, p. 8) señalan que: "La escuela, como institución comunitaria que es, debe ampliar sus cometidos educativos, culturales y sociales, diversificando su presencia en el escenario local, asumiendo para ello la puesta en marcha de dinámicas y proyectos que la reafirmen como un agente destacado en la construcción de la sociedad educadora.”

No obstante, aunque actualmente es común considerar la necesidad del trabajo conjunto y coordinado de los diferentes agentes que tienen alguna incidencia educativa sobre una comunidad, frecuentemente este planteamiento es aún hoy más teórico que real (Longás, Civís, Riera, Fontanet, Longás y Andrés, 2008). Estos autores destacan algunas experiencias internacionales que, desde una perspectiva comunitaria, buscan nuevas respuestas a los retos educativos actuales. Tal es el caso de la Asociación Internacional de Ciudades Educadoras (AICE), los Projet 
Éducatif Local y los Contrat Éducatif Local franceses y las Education Action Zones del Reino Unido. También mencionan algunos proyectos que se llevan a cabo en España, que parten de las necesidades y recursos locales y persiguen impulsar contextos sociales más educativos y el trabajo coordinado de agentes que inciden sobre la comunidad, de una u otra manera: las ciudades educadoras, los proyectos educativos de ciudad o las acciones educativas integradas. Son más frecuentes en secundaria y el origen de estas experiencias se encuentra, principalmente, en la búsqueda de mejorar el fracaso escolar y construir una red local que responda a sus necesidades.

Todo esto nos lleva a afirmar que es urgente que la escuela se reubique para responder a las demandas sociales actuales, y participe en procesos comunitarios que incluyan diferentes miembros de la comunidad y agentes sociales externos, además de garantizar la igualdad e impulsar iniciativas de implicación personal, movilización colectiva, mejora de las condiciones de vida, respeto a la identidad cultural de cada individuo y tolerancia a las propuestas del resto (Cieza, 2006). Asimismo, será fundamental realizar programas de intervención comunitaria en los que la comunidad y la escuela sean sujeto y objeto del desarrollo, que dinamicen los recursos comunitarios tomando como punto de partida un contexto y fomenten la participación, el trabajo en equipo, la implicación, la iniciativa, el respeto y el diálogo para adoptar una actitud reflexiva y crítica que favorezca el enriquecimiento personal de todos los agentes que tomen parte, fuera y dentro de la escuela (Cieza, 2006; Villena y Molina, 2015).

En este sentido, cabe señalar que la expresión artística del alumnado (musical, corporal, etc.) se convierte en un vehículo especialmente idóneo para la conexión y el entendimiento, ya que forma parte de la dimensión comunicativa de las personas y sociedades y constituye un elemento importante en la construcción de su identidad (Cabedo, 2014a). Especialmente a través de la participación comunitaria, que constituirá, por tanto, uno de los pilares del programa objeto de este estudio, la expresión artística del alumnado facilita la creación de espacios de participación, integración y democracia cultural en las sociedades (Cabedo, 2014b). Una vez más, queda reflejada la necesidad de conocer el contexto donde se ubican estos espacios de integración en los que se realizan las actividades de música comunitaria.

Asimismo, debemos destacar la importancia de la participación en el desarrollo de cualquier programa comunitario, precedida del esfuerzo por escuchar y analizar el entorno, el contexto donde se va a transitar (Fernández, 2016). Así, se hace evidente, por todo lo expuesto, que la comunidad no puede ser obviada en el análisis de la vida escolar donde se inserta la escuela, por lo que el diagnóstico de la vida y relaciones comunitarias se hace necesario. Uno de los principales retos, por tanto, de este estudio, será seleccionar los elementos de análisis más adecuados para el diseño de un programa que fomente la convivencia positiva, el entendimiento cultural y la conexión con la comunidad escolar y de la realidad que la envuelve.

\section{IMPORTANCIA DE LA COMUNIDAD EN LA EDUCACIÓN Y EN EL APRENDIZAJE-SERVICIO}

Su desarrollo ha encarnado durante más de 40 años una constante innovación que aspira a un cambio en las formas de enseñanza tradicionales, sobrepasando los límites del currículo para permitir el desarrollo de nuevas formas de acción ciudadana organizada que permitan co-construir espacios sociales y educativos (Opazo y Aramburuzabala, 2019). 
El ApS genera oportunidades para que el alumnado se enfrente a sus propias creencias y prejuicios (Peralta, O’Connor, Cotton y Bennie, 2016), siendo numerosos los estudios que concluyen que es un enfoque metodológico que promueve tanto la inclusión (Carrington, Mercer, Iyer y Selva, 2015; Carrington y Saggers; 2008; Lleixà y Nieva, 2018; O’Meara, Huber y Sanmiguel, 2018), como la conciencia sobre la diversidad (Cámara, Díaz y Ortega, 2017; Mergler, Carrington, Kimber, Bland y Boman, 2017) y la multiculturalidad (Chang, et al., 2011; Peralta et al., 2016). Sin ir más lejos, los contextos multiculturales representan un escenario de aplicación del ApS muy concurrido (Peralta, et al., 2016), en tanto que ayudan a incrementar la comprensión del alumnado sobre dicha realidad (Chang, Anagnostopoulos y Omae, 2011; Palpacuer-Lee y Curtis, 2017). En este sentido, parece conveniente abrir la institución escolar al entorno social, en respuesta a un cambio de paradigma que inste a trabajar con el alumnado en una realidad que trascienda del aula.

En el nivel educativo que nos ocupa por fin empieza a emerger con fuerza el ApS (Chiva-Bartoll, Salvador-García, Ferrando-Félix, \& Cabedo-Mas, 2019), dado que se trata de "una estrategia de bajo coste que puede beneficiar a los estudiantes de primaria cuando se implementa correctamente" (Fair y Delaplane, 2015, p. 19). La escuela, como institución comunitaria que es, debe ampliar sus cometidos educativos, culturales y sociales, teniendo presencia en el contexto social y apostando decididamente por dinámicas y proyectos que la reafirmen como un agente destacado en la construcción de la sociedad (Caballo y Gradaíle, 2008). En este marco, la meta del ApS radica en conocer y aprovechar las posibilidades y recursos del entorno para participar, en la medida de lo posible, en su mejora y desarrollo. Además, aunque desde el paradigma educativo actual no es fácil que el alumnado interactúe con actores y recursos externos, ni mucho menos fuera de los muros escolares, el ApS abre la puerta a un contacto más directo entre el alumnado y los miembros de la comunidad, apostando por un aprendizaje que, por definición, se adquiere a través del servicio prestado (Billig, 2002; Williams, 2016).

Así mismo, además de los beneficios para el alumnado, las investigaciones realizadas también aluden a ventajas para las escuelas participantes (Billig, 2002). Por ejemplo, Kielsmeier (2003) apunta a que tanto el clima escolar como la visión que tiene la comunidad sobre el alumnado son cuestiones que se ven afectadas positivamente por el ApS. Del mismo modo, la percepción que tiene el alumnado sobre la escuela también mejora (Scales, et al., 2000); mientras que las escuelas refieren impactos significativos en el compromiso y el aprendizaje de los estudiantes con la comunidad (Fair y Delaplane, 2015). Además, parece que el ApS, en el mismo acto de inmiscuir al alumnado en la construcción de su propia realidad social (Carter, 1997), lo lleva a desarrollar y/o reforzar su sentido de pertenencia a un lugar y comunidad (Williams, 2016), potenciando la percepción de que está en su mano ejercer un impacto valioso para su propia comunidad (Billig, 2000).

Sin embargo, el mero contacto o interacción entre los agentes que intervienen en el ApS no garantiza que se produzca una comprensión consciente del alumnado sobre la condición de vulnerabilidad social del entorno (Palpacuer-Lee y Curtis, 2017). Es por este motivo por el que resulta necesario incorporar elementos de reflexión y análisis en las fases iniciales del ApS, a fin de comprender mejor las raíces de las necesidades afrontadas (Chiva-Bartoll, Peris, \& Piquer, 2018. 
En este sentido, no cabe duda de que para acometer con cierta garantía de éxito la planificación del ApS, es necesario partir de un riguroso diagnóstico del contexto y de las necesidades sobre las que se prevé actuar. Sin duda, en el ApS el contexto es la piedra angular del proceso de enseñanza-aprendizaje, por lo que sin un análisis inicial del mismo parece imposible orientar con la necesaria precisión la hoja de ruta a seguir. Por tanto, el presente artículo tiene por objetivo mostrar la fase de diagnóstico de un barrio donde se aplica un programa de ApS y las aportaciones que suponen en el programa concreto. El objetivo específico de este artículo es desarrollar y ejemplificar las diferentes fases que se suceden a la hora de llevar a cabo el análisis del barrio en el que está inmerso un centro educativo, en este caso una escuela.

\section{EL CONTEXTO EN EL CASO DE UN PROGRAMA CONCRETO: MUSIQUEM}

De acuerdo con lo expuesto, se propone desarrollar el programa Musiquem! Fent música comunitària a les escoles [en castellano, ¡Musiquemos! Haciendo música comunitaria en las escuelas] en el CEIP Mestre Canós Sanmartín de la localidad de Castelló de la Plana, con el fin principal de aprovechar las posibilidades de la expresión musical y corporal como elementos de comunicación, de convivencia positiva y entendimiento intercultural a través de proyectos de Aps, fomentando la conexión con la comunidad escolar y con la realidad que envuelve al centro. Musiquem propone un trabajo de colaboración entre docentes de educación artística, musical y corporal y artistas residentes en el aula, que trabajarán con proyectos de ApS y actividades musicales comunitarias dentro y fuera del centro, fomentando la conexión con la comunidad escolar y la realidad que lo envuelve. Se lleva a cabo dentro de las horas lectivas, permitiendo la participación de todo el alumnado.

El programa Musiquem se articula a través de proyectos de ApS con objeto de fomentar la cooperación entre estudiantes y miembros de la comunidad próxima al centro escolar. Se pretende involucrar al alumnado en actividades de servicio a la sociedad y proporcionarles una experiencia de aprendizaje motivadora para su desarrollo personal y académico. El diseño incluye actuaciones artísticas en centros de adultos mayores, centros de día, centros de salud y asociaciones vecinales próximas al centro educativo.

Musiquem parte de una iniciativa de los departamentos de Educación y Pedagogía de la Universitat Jaume I en el marco de un proyecto de investigación financiado por el Ministerio de Ciencia, Innovación y Universidades (referencia EDU2017-86311-P). Cuenta también con el apoyo del Ayuntamiento de Castelló, quien financia algunas de las acciones relacionadas con la dimensión pedagógica del mismo.

La complejidad de la elaboración de un programa de esta índole, que pretende la participación y fluida relación entre los miembros de la comunidad educativa (equipo docente del centro que imparte en los grupos que participan en el proyecto, equipo directivo del centro, padres y madres del alumnado, asociaciones del barrio...), hace que sea necesario un diagnóstico previo que ayude a prevenir deficiencias metodológicas significativas que se han detectado en muchos de estos programas (Rodríguez, \& Cabedo, 2017). Nuestra contribución consiste en presentar los instrumentos que han propiciado este diagnóstico, así como las aportaciones que han supuesto a nuestro programa. 


\section{INSTRUMENTOS DE RECOGIDA DE INFORMACIÓN: ELABORANDO EL DIAGNÓSTICO DEL BARRIO}

Para realizar el diagnóstico del barrio en el cual está inmerso un centro educativo es necesario contar con diferentes instrumentos de recogida de información de todos los agentes que conforman la comunidad educativa. En muchas ocasiones, como veremos, los instrumentos de recogida de información pueden ser empleados también como recurso didáctico. Este hecho puede constatarse en el ejemplo de la maqueta y las grabaciones que se describen acto seguido.

En relación a los criterios de selección de los instrumentos, así como a la validez de los mismos, dado que el interés de la propuesta se centra en una experiencia y un contexto específicos, nuestra intención no aspira en ningún momento a incitar el uso de una serie de instrumentos de forma categórica o generalizada, sino a relatar cómo su elección debe ajustarse a cada caso y situación particular (Flyvbjerg, 2006). Por tanto, desde un enfoque intencional ecológico, la elección de los instrumentos emergió de un proceso subjetivo de discusión llevado a cabo por el grupo de expertos responsables del proyecto. El debate, llevado a cabo por un equipo de 6 personas doctoras con experiencia docente y formadas en el ámbito de la intervención educativa socio-comunitaria, se centró en torno a criterios como: efectividad, aplicabilidad, accesibilidad y potencial didáctico (siempre que fuera posible) de los instrumentos valorados. En todo momento se tuvo presente que la transferibilidad, desde este enfoque metodológico centrado en un caso concreto, se logra cuando los lectores perciben un notable grado de superposición con su propia situación, lo que puede llevarlos a transferir los hallazgos o resultados a sus propias situaciones contextuales (Tracy, 2010; Hodge y Sharp, 2016).

A continuación, describimos aquellos que hemos utilizado en nuestro proyecto, presentando ejemplos de cada uno de ellos y apuntando algunas de las acciones que se han realizado a partir de los resultados obtenidos con objeto de facilitar su comprensión.

\subsection{Maqueta en relieve de la escuela y del barrio}

La maqueta es un instrumento didáctico que ha sido empleado exitosamente en diferentes contextos educativos (Albarán-Torres, Urrutia-Martínez, Ibarra-Peso, Miranda-Díaz \& Mesa-Vázquez, 2018; Ortiz de Zárate Arriola, 2014). En el proyecto que desarrollamos, la maqueta permite la representación de espacios de la superficie terrestre a diferente escala de manera tridimensional. Con este material se pretende visibilizar la escuela y su entorno. Para ello, se utilizaron diferentes colores que representaban los espacios que componían el barrio, estos son: asociaciones, plazas y parques, centros de enseñanza, centros de promoción de actividad física y deporte, administración y servicios públicos y arte (música, danza, teatro y museos). La maqueta fue construida por el propio alumnado y fueron ellos y ellas los encargados de ir ubicando dichos espacios en la maqueta. El trabajo de este instrumento presentaba un doble objetivo: por una parte, conseguir tener un conocimiento más profundo de los lugares y recursos que rodean a la escuela tanto para el alumnado, profesorado y comunidad educativa en general, y por otra como material didáctico a utilizar en las diferentes materias. En las siguientes imágenes se puede apreciar el primer diseño de la maqueta y la maqueta una vez finalizada. Figura 1. 

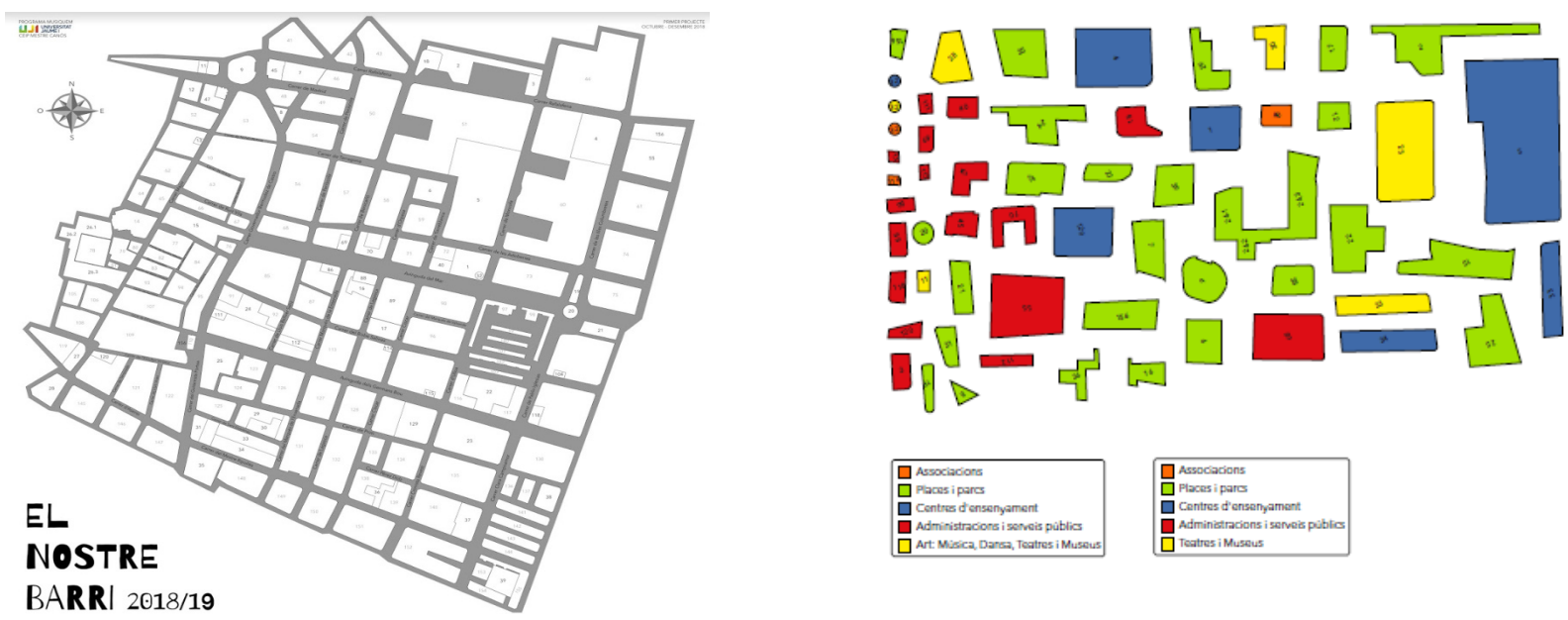

Figura 1. Diseño de la maqueta y maqueta del barrio. Fuente. Elaboración propia.

\subsection{Grabaciones sonoras del barrio}

A partir del desarrollo de la maqueta y su estudio a través de diferentes actividades en el aula pudimos establecer un mapa social de los espacios en los que nuestros estudiantes se mueven de manera habitual y, consecuentemente, conocen. En este proceso, descubrimos que los estudiantes de la escuela tienen un margen de movimiento reducido en el barrio, y hacen un uso muy limitado de los recursos sociales (asociaciones, espacios de ocio, etc.) que el barrio ofrece. En este contexto, decidimos diseñar acciones para promover el conocimiento de los espacios existentes en su barrio. Para ello, invitando a las familias de los estudiantes, propusimos investigaciones sobre los diferentes espacios y salidas a estos lugares en los que, junto con una visita a cada espacio, realizamos grabaciones de paisajes sonoros del espacio. Estas grabaciones sonoras son material para el diseño de actividades como escuchas activas y debates sobre las sensaciones que emergen de dicha audición, recursos para futuras composiciones musicales, etc. En definitiva, estas grabaciones sonoras permiten que el estudiantado conozca su barrio desde más allá del sentido de la vista, desarrollando nuevos y diferentes vínculos con los espacios que les rodean. Estas acciones estuvieron fuertemente vinculadas con el siguiente instrumento que comentamos.

\subsection{Dosier del alumnado: descubrimos nuestro barrio}

La maqueta fue un material complementario y necesario para poder realizar este instrumento. Desde el proyecto se creó un dossier que contenía una serie de cuestiones y que anexado tenía la imagen de la maqueta en tamaño A3. El alumnado, junto a sus familias y aprovechando un periodo vacacional, debía indagar sobre uno de los espacios de la maqueta, a cada uno de los y las alumnas se le asignó un lugar y debían explorarlo a partir de las siguientes preguntas: ¿Cómo se llama tu espacio?; ¿Cuál es la dirección de tu espacio?; Describe brevemente tu espacio. ¿Tiene un horario de uso o atención al público?; ¿Cuál es su horario?; ¿Quéfunción tiene tu espacio?; ¿Qué personas 
aprovechan más este espacio?; ¿Qué sentiste cuando fuiste a visitar el espacio?; ¿Te gustó la sensación?; ¿Habias visitado este edificio anteriormente?; ¿Fuiste solo o acompañado?; ¿Tus padres conocían el edificio?; ¿Conoces a alguien más que te pueda dar información sobre el espacio visitado?; ¿Quién?; ¿Qué información?; ¿Qué otros edificios interesantes descubriste de camino cuando fuiste a ver a tu edificio?

Como se puede observar, con estas cuestiones se pretendía tener una información pormenorizada sobre el espacio, pero también sobre las sensaciones que le evocaban al alumnado. Como hemos mencionado el dossier tenía anexado el mapa de la maqueta en A3 y en él debían señalar el espacio y la distancia de este a la escuela. Conviene decir que fue una actividad que tenía como objetivo no solo conocer el propio edificio asignado, sino también el resto del barrio tanto por parte del alumnado como de las familias. A continuación, presentamos un extracto de dossier empleado: Figura 2.

Conviene destacar que, una vez finalizada la actividad y previo análisis de los dossiers se llevaron a cabo grupos de discusión con parte del alumnado para poder recabar información más detallada de todos los elementos que convenimos necesarios para nuestra investigación.

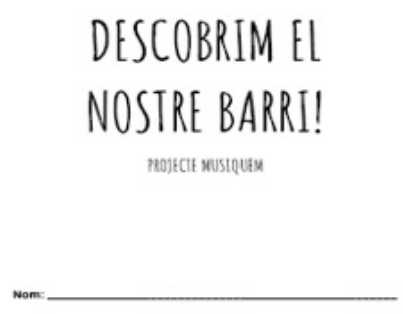

QUUE ES MUSIQUEM?

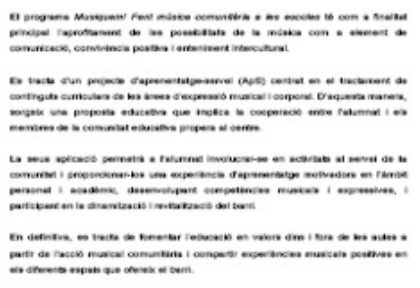

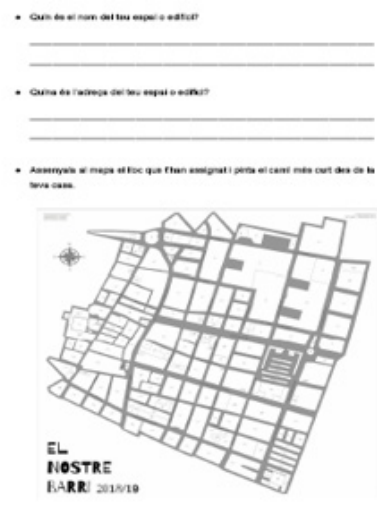

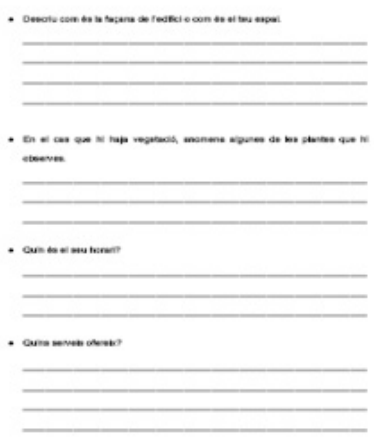

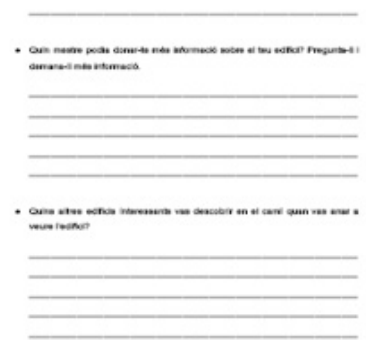

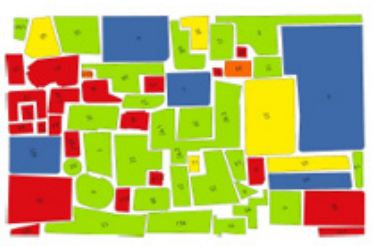

Imagen 2. Dosier del alumnado: descubrimos nuestro barrio. Fuente. Elaboración propia 


\subsection{Ficha para las familias}

Las familias son otro de los elementos necesarios para poder obtener información relevante sobre el barrio. Para conocer cuáles eran sus percepciones y opiniones, tanto del centro como del barrio, se acordó elaborar una ficha de fácil respuesta y entregarla a través de sus hijos e hijas. Las preguntas que conformaron dicho documento relativas al barrio fueron las siguientes: ¿Su hijo/a participa en actividades extraescolares en el centro o fuera de él?; ¿En cuáles?; ¿Su hijo/a participa en alguna actividad cultural?; ¿En cuál?; ¿Viven en el barrio?; ¿Conocen el barrio?; ¿Se sienten del barrio?; ¿Por qué?; ¿Tienen amigos/as en el barrio (referente a ustedes como padres)?; ¿Conocen asociaciones del barrio?;¿Cuáles?; ¿Utiliza las asociaciones/organizaciones/espacios del barrio (tanto ustedes como padres como sus hijos/as?).

Esta primera recogida de información nos podría servir para hacernos una idea global sobre la imagen que tienen las familias del barrio y el uso que hacen de sus recursos (si es que los hay), entre otros. Del mismo modo que con el alumnado, consideramos necesario realizar un grupo de discusión posteriormente para poder abordar con detenimiento aquellos aspectos surgidos y que son de interés para tener un conocimiento más profundo de la escuela y de su entorno.

A raíz de estas interacciones con las familias, descubrimos que muchas de ellas no se sienten enraizadas en el barrio: muchas son familias que han inmigrado a la ciudad recientemente y no han construido fuertes vínculos con el contexto que les rodea; otros llevan tiempo en el barrio, pero no siempre conocen los recursos que el barrio ofrece. Por otra parte, aprovechamos esta coyuntura para buscar una mayor implicación de las familias en el proyecto Musiquem, indagando acerca de las posibles maneras en las que padres, madres y familiares se verían cómodos participando y tomando decisiones en el proyecto de centro.

Como consecuencia de este diagnóstico, se diseñará un proyecto enfocado a establecer vínculos entre conjuntos instrumentales y vocales formados por estudiantes del centro y por sus familiares. De este modo, se crearán conjuntos corales con padres, madres y/u otros familiares del centro que, con metodologías similares a las del proyecto Musiquem, entren a formar parte de las acciones llevadas a cabo por el centro y los investigadores. En horario extraescolar, aquellas familias que lo desean pueden disfrutar de una hora semanal de taller de canto coral al tiempo que sus hijos e hijas reciben lecciones de refuerzo en la práctica instrumental (violín o violoncello). Se diseñarán en este marco actividades y conciertos conjuntamente entre la orquesta de niños y niñas y el coro de familias.

\subsection{Autoinforme para los y las maestras participantes en el proyecto}

El último [4] de los pilares que consideramos que aporta información sobre el barrio en el que está inmersa la escuela es su profesorado. Es de vital importancia que los y las docentes conozcan la realidad inmediata de sus centros para comprender de una forma clara el contexto y así adaptar las intervenciones que realicen en sus aulas y en la escuela. El instrumento que se utilizó fue el autoinforme, donde el profesorado libremente debía expresar su opinión sobre su pasado y futuro en el centro, dando respuestas a cuestiones personales como: ¿Por qué eligió este centro?; ¿Quéfue lo más importante para usted a la hora de elegir este centro?; ¿Se ve en el centro a largo plazo? 
Así como a preguntas sobre la imagen de centro: ¿Cómo lo describiría?; ¿Cuáles son los puntos fuertes y débiles de la escuela desde su visión?; ¿Cómo creen que lo ven las familias?; ¿Cómo cree que lo ven las personas del barrio?; ¿Cómo creen que lo ven las personas ajenas al centro y al barrio?).

Y también sobre sus conocimientos acerca del barrio donde se ubica el centro (asociaciones, realidad del barrio...).

Del mismo modo que con las familias, esta primera información nos sirvió para poder realizar posteriormente entrevistas en profundidad y abordar cuestiones que no hayan quedado claras a través de los autoinformes. La información recabada en estas entrevistas, a su vez, nos permitió complementar la información inicial con algunos detalles y especificidades, aunque no implicó modificaciones substanciales o de carácter estructural para el diseño del proyecto de ApS.

\subsection{Entrevistas agentes del barrio}

Un elemento esencial que conforma cualquier barrio son las personas que viven en él. En este caso, para conocer qué personas podrían dar información valiosa sobre el barrio y la escuela se preguntó al equipo directivo de la escuela y al alumnado de las tres clases que participaban en el proyecto sobre qué agentes consideraban eran importantes y formaban parte del día a día del barrio. Las respuestas fueron coincidentes y se procedió a realizar las entrevistas pertinentes con una serie de preguntas básicas: ¿Cuántos colegios forman parte del barrio?; ¿A qué colegio lleva a sus hijos/as? (En el caso que no tenga hijos a qué colegio le gustaría llevarlos); ¿Cuál es la imagen que tiene de la escuela?; Aspectos positivos, aspectos negativos, propuestas de mejora. ¿Cuál es la imagen que tiene del barrio en el que está inmerso el centro?; ¿Cómo es la convivencia del barrio?; ¿Existe mucha diversidad?

Con estas entrevistas se pretendía conocer cuál era la imagen que las personas que viven en los alrededores del barrio y de la escuela. Se quería dar un paso más y abordar un tema importante como es la convivencia de este y quienes mejor para dar este tipo de información que la persona que regenta el quiosco, el farmacéutico, el policía de la tendencia alcaldía o la directora de la asociación de mayores.

A continuación, presentamos un extracto de una de las entrevistas:

\section{Entrevistadora: ¿Cómo es la imagen que tiene del barrio?}

Agente: Se trata de un barrio abierto, con muchas posibilidades. Hay muchos servicios para las personas mayoresy es muy cómodo para vivir en referencia al transporte. Además, hay muchos servicios administrativos y es muy céntrico. Siempre hay aspectos que se pueden mejorar, pero, en general, me gusta el barrio.

Entrevistadora: ¿Cómo es la convivencia en el barrio? ¿Existe much a diversidad?

La convivencia entre los vecinos es tranquila. Somos gente de barrio, sobre todo, personas mayores e inmigrantes. Existe mucha diversidad, pero muy bien integrada y sin conflictos importantes. 


\subsection{Trabajo colaborativo con las asociaciones}

A raíz del análisis de la información recabada por todos estos instrumentos advertimos que tanto la comunidad escolar (estudiantado, profesorado y familias) como los habitantes del barrio no tienen gran conocimiento de la historia del barrio y el recorrido cultural y social que ha llevado a que el barrio hoy tenga unas determinadas características. Sentimos necesario que nuestros estudiantes no perdiesen de vista la importancia de la historia también en la cotidianidad y, por esta razón, propusimos actividades en conjunto con la asociación de personas mayores del barrio (Centro Especial de Asistencia al Mayor - CEAM).

Con este propósito, a través de esta asociación, contactamos con personas mayores que vivían en el barrio y que habían nacido y crecido en él. De este modo, nuestros estudiantes iniciaron un proceso de investigación centrado en conocer la historia del barrio y, posteriormente, programamos una serie de conferencias a partir de las cuales nuestros estudiantes tuvieron la posibilidad de conocer de primera mano esas narrativas de la historia, y formular una serie de interrogantes.

A raíz de esta experiencia, la asociación de personas mayores quiso también invitar al alumnado de la escuela a conocer su centro y, en este contexto, se programó un concierto conjunto en el que participaron el coro de personas mayores, la orquesta de plectro de la asociación y Musiquem.

\section{PROGRAMA DE APRENDIZAJE-SERVICIO IMPLEMENTADO Y REFLEXIONES FINALES}

De acuerdo con el propósito principal de este trabajo, podemos extraer algunas ideas derivadas de la reflexión sobre los materiales trabajados. El ApS constituye una vía metodológica que abre las puertas del espacio escolar a la sociedad, fomenta la participación local e implica la participación de todos los miembros de la comunidad social. No obstante, las diferencias y necesidades de las instituciones educativas implicadas y el lugar donde se insertan exigen un análisis previo del contexto que nos permita adaptar el programa que se va a implantar.

En el caso de esta experiencia, del diagnóstico recién descrito emergió un programa de ApS que, en base a la información de los instrumentos recién descritos, consistió en las siguientes actuaciones:

Inicialmente, se llevó a cabo una etapa enfocada en establecer el contacto inicial de los estudiantes con problemas específicos de la comunidad, como la falta de sentido de pertenencia a la comunidad y conocimiento sobre los edificios y espacios culturales que conforman el barrio y las actividades culturales (información que pudimos conocer a través de las grabaciones sonoras y del dosier del alumnado fundamentalmente). Esta fase se llevó a cabo a través de salidas del centro para visitar colectivos y asociaciones culturales del barrio con el fin de conocer y profundizar sobre las posibilidades de interacción sociocultural del barrio (empleando aquí la información recabada tanto en la maqueta, como en el trabajo colaborativo con las asociaciones y a partir de las entrevistas con los agentes del barrio). Después de esta fase vino una etapa de reflexión guiada y pensamiento crítico, en la que los estudiantes tuvieron que enfocarse en contrastar opiniones y proponer un plan de acción para trasladar lo que estaban aprendiendo a las familias, basado en las materias académicas involucradas (esta actividad tuvo en cuenta la información 
de los autoinformes de los maestros, sus intereses y conocimiento del centro y su entorno, etc.). Además, la reflexión se extendió a lo largo del programa, en tanto que se pidió al alumnado que escribiera un diario de seguimiento reflexivo sobre las tareas y vivencias experimentadas. Cada alumno y alumna tuvo que reflexionar sobre sus experiencias, posibles problemas detectados y sus soluciones, así como sobre los principales aprendizajes adquiridos, etc. Finalmente, la ejecución de las tareas constituía una fase de experimentación en la que el alumnado profundizó sobre las oportunidades culturales y sociales del barrio a través de nuevas salidas y visitas a asociaciones y lugares importantes del barrio (conservatorio, museo, clubes deportivos, auditorio, etc.). Esta última fase fue posible gracias a la información recabada por el conjunto de los instrumentos empleados.

Después de esta experiencia, podemos confirmar que los elementos utilizados que hemos descrito en el presente artículo nos han permitido realizar el diagnóstico del barrio en el que se inserta el centro escolar, lo que también ha facilitado el diseño de las estrategias principales del programa. Así, detectar la falta de arraigo de algunas familias nos ha llevado a proponer actividades para su integración en la comunidad escolar través de la formación de un coro de familias; la realización de la maqueta nos ha permitido constatar los lugares que conocen y cuáles les son ajenos, para lo que se han programado tertulias sobre el barrio y salidas para conocer determinados lugares. Asimismo, tras las entrevistas a los agentes del barrio, se han programado conciertos en determinados lugares para visibilizar el proyecto y el centro, los estudiantes llevaron a cabo actividades en público de percusión corporal, conciertos musicales, etc. Asimismo, tras las entrevistas a los agentes del barrio se han programado conciertos en determinados lugares para visibilizar el proyecto y el centro.

Por tanto, constatamos que los instrumentos utilizados proporcionan la oportunidad de ajustarse en mayor medida a la realidad, las demandas y necesidades de la comunidad y de las personas que forman parte de esta facilitan la identificación de la comunidad con el proyecto Musiquem. Y que lo sientan como propio, a fin de promover a través de este una cultura colaborativa entre la escuela y la comunidad. Igualmente, se espera que permita crear una primera implicación con el barrio, generar una motivación y actitud positiva hacia las acciones que se van a realizar e incluso descubrir cuáles podrían ser los posibles recursos (Cieza, 2006). Esto supone un proceso de sensibilización en el contexto general, tanto en la comunidad escolar como en el barrio-contexto donde ésta se inserta, que implica un mayor número de posibilidades para profundizar en el conocimiento mutuo para evolucionar, cambiar, mejorar y transformar (Cieza, 2006).

Por otra parte, posibilita ser consciente de las necesidades o intereses comunes, lo que facilita, a su vez, el diseño de estrategias y actividades orientadas a un proceso integrador entre los agentes sociales que viven y transitan el barrio, vinculadas a su vez con iniciativas de ApS en las que se refuerza el aprendizaje adquirido a través del servicio prestado en contacto directo entre la escuela o la comunidad.

Por último, un diagnóstico realizado con los instrumentos señalados permite reflexionar sobre el papel de la escuela en el barrio y viceversa, y avanzar en la conexión entre ambos, ya que los procesos de mejora escolar están estrechamente vinculados con la comunidad social, y la mejora de la sociedad está relacionada directamente con la mejora educativa. Es preciso subra- 
yar que el proceso de cambio y mejora que requiere la educación está vinculado inevitablemente con la participación de todos los agentes interesados en este proceso, lo que incluye no sólo a los profesionales de la educación, sino también al alumnado y sus familias y a todos los miembros de la comunidad social y educativa (Parrilla, Muñoz y Sierra, 2014).

\section{REFERENCIAS}

Albarrán Torres, F., et al. (2018). Maquetas como estrategia didáctica en estudiantes dela salud.Educación Médica. 2018. DOI: http://dx.doi.org/10.1016/j.edumed.2018.08.003.

Billig, Shelley H. (2002). Support for K-12 service learning practice: A brief review of the research. Educational Horizons. 80,184-189. https://bit.ly/2Kk5R4v

Bringle, R., y Hatcher, J. (1995). A service-learning curriculum for faculty. The Michigan Journal of Community Service-Learning. 2, 112-122. https://bit.ly/2WrNpJz

Caballo, B. \& Gradaílle, R. (2008). La educación social como práctica mediadora en las relaciones escuela-comunidad local. Pedagogía Social. Revista Interuniversitaria. (15), 45-55. DOI: https://doi. org/10.7179/PSRI_2008.15.04

Cabedo, A. (2014a). La música como proyecto socioeducativo. In A. Giráldez, Didáctica de la música en primaria (pp. 101-122). Madrid: Síntesis.

Cabedo, A. (2014b). La música comunitaria como modelo de educación, participación e integración social. Eufonía: didáctica de la música. 60,15-23.

Cámara, Á., Díaz, E. y Ortega, J. (2017). Aprendizaje servicio en la universidad: ayudando a la escuela a atender la diversidad a través de las TIC. Bordón. Revista de Pedagogía. 69(3), 73-87. DOI: http:// dx.doi.org/10.13042/Bordon.2017.51320.

Carrington, S. y Saggers, B. (2008). Service-learning informing the development of an inclusive ethical framework for beginning teachers. Teaching and Teacher Education. 24(3), 795-806. DOI: http:/l dx.doi.org/10.1016/j.tate.2007.09.006.

Carrington, S., Mercer, K. L., Iyer, R. y Selva, G. (2015). The impact of transformative learning in a critical service-learning program on teacher development. Reflective Practice.16(1), 61-72. DOI: http://dx.doi. org/10.1080/14623943.2014.969696.

Carter, G. R. (1997). Service-learning in curriculum reform. In Schine, J. (Ed.), Service learning: Ninetysixth year book of the National Society for the Study of Education, Part I (pp. 69-78). Chicago: University of Chicago Press.

Ceballos, E. (2006). Dimesiones de análisis del diagnóstico en educación: El diagnóstico del contexto familiar. RELIEVE. V. 12 (1), 33-47. (Recuperado de https://bit.ly/2HHSpWi).

Chang, S., Anagnostopoulos, D. y Omae, H. (2011). The multidimensionality of multicultural service learning. Teaching and Teacher Education. 27(7), 1078-1089. DOI: http://dx.doi.org/10.1016/j. tate.2011.05.004.

Chiva-Bartoll, Ó., Peris, C. C., \& Piquer, M. P. (2018). Investigación-acción sobre un programa de aprendizaje-servicio en la didáctica de la educación física. Revista de investigación educativa, 36(1), 277-293. 
Chiva-Bartoll, O., Santos-Pastor, M. L., Martínez-Muñoz, F. \& Salvador-García, C. (2019). Valoración del impacto del aprendizaje- servicio universitario en el ámbito de la actividad física y el deporte: una propuesta desde la teoría de stakeholders. Publicaciones, 49(4), 27-46

Chiva-Bartoll, O., Salvador-García, C., Ferrando-Félix, S., \& Cabedo-Mas, A. (2019). Aprendizaje-servicio en educación musical: revisión de la literatura y recomendaciones para la práctica. Revista Electrónica Complutense de Investigación en Educación Musical.16, 57-74.

Cieza-García, J. A. (2006). Educación comunitaria. Revista de educación. 339, 765-799.

Deeley, S. (2016). El Aprendizaje Servicio en educación superior. Teoría, práctica y perspectiva crítica. Madrid: Narcea. https://bit.ly/3p6Za4G

Fair, Cintya. D., \& Delaplane, E. (2015). It is good to spend time with older adults. You can teach them, they can teach you: Second grade students reflect on intergenerational service learning. Early Childhood Education Journal. 43(1), 19-26. DOI: http://dx.doi.org/10.1007/s10643-014-0634-9.

Fernández, E. (2016). Habitar las ciudades a través de metodologías participativas y procesos de transformación social: pedagogías de la democracia ausente y recuperación del espacio público. En Villena J.L. \& Molina E. (2016). Ciudades con vida: Infancia, participación y movilidad (pp. 69-102). Barcelona: Grao.

Flyvbjerg, B. (2006). Five misunderstandings about case-study research. Qualitative Inquiry. 12, 219-245. DOI: https://doi.org/10.1177/1077800405284363

Hodge, K., and Sharp, L. A. (2016). Case studies. En Smith, B. \& Sparkes A. (Eds.). Routledge handbook of qualitative research in sport and exercise (pp. 62-75). New York: Routledge. https://bit.ly/3akGc6p

Kielsmeier, J. (2003). A time to serve, a time to learn: New roles for youth. Generator. 21(3), 5-14.

Lleixà, T. y Nieva, C. (2018). The social inclusion of immigrant girls in and through physical education. Perceptions and decisions of physical education teachers, Sport, Education and Society. DOI:10.1080/1357 3322.2018.1563882.

Longás, J., Civís, M., Riera, J., Fontanet, A., Longás, E., \& Andrés, T. (2008). Escuela, educación y territorio. La organización en red local como estructura innovadora de atención a las necesidades socioeducativas de una comunidad. Pedagogía Social. Revista Interuniversitaria. (15), 137-151. DOI: https://doi.org/10.7179/PSRI_2008.15.11.

Mergler, A., Carrington, S., Kimber, M., Bland, D. y Boman, P. (2017). Exploring the Value of ServiceLearning on Preservice Teachers. Australian Journal of Teacher Education. 42(6), 69-80. DOI: http:// dx.doi.org/10.14221/ajte.2017v42n6.5.

O'Meara, J., Huber, T. y Sanmiguel, E. (2018). The role of teacher educators in developing and disseminating global citizenship education strategies in and beyond US learning environments. Journal of Education for Teaching. Epub. DOI:10.1080/02607476.2018.1516347

Opazo, H. y Aramburuzabala, P. (2019). Service Learning in Teacher Training - A Case Study of the Autonomous University of Madrid. En P. Aramburuzabala, L. Mcllrath y H. Opazo (eds.), Embedding Service-Learning in European Higher Education (pp. 154-165). Londres: Routledge. DOI: https://doi. org/10.4324/9781315109053

Ortiz de Zárate Arriola, E. (2014). La maqueta como un recurso educativo para una didáctica del entorno urbano en la educación secundaria obligatoria. (Tesis Fin de Máster). Universidad Internacional de la Rioja, La Rioja. (Recuperado de https://bit.ly/30sFHkT). 
Palpacuer-Lee, C. y Curtis, J. H. (2017). Into the Realm of the Politically Incorrect: Intercultural Encounters in a Service-Learning Program. International Journal of Multicultural Education. 19(2), 163-181. DOI: http://dx.doi.org/10.18251/ijme.v19i2.1239.

Parrilla Latas, Á., Muñoz-Cadavid, M. A., \& Sierra Martínez, S. (2014). Proyectos educativos con vocación comunitaria. Revista de Investigación en Educación, 11(3) pp. 15-31

Peralta, L., O'Connor, D., Cotton, W. y Bennie, A. (2016). Pre-service physical education teachers' indigenous knowledge, cultural competency and pedagogy. Teaching Education. 27(3), 248-266. DOI: http://dx.doi.org/10.1080/10476210.2015.1113248.

Rodríguez, A., \& Cabedo, A. (2017). Espacios musicales colectivos durante y después del conflicto armado como lugares de preservación del tejido social. Revista Co-herencia Universidad EAFITDepartamento de Humanidades, 14(26).

Sales Ciges, A. y Garcia López, R. (1997). Programas de Educación Intercultural. Bilbao: Desclée De Brouwer.

Scales, Peter. C., Blyth, Dale. A., Berkas, Thomas. H., \& Kielsmeier, James. C. (2000). The effects of servicelearning on middle school students' social responsibility and academic success. The Journal of Early Adolescence. 20(3), 332-358. DOI: https://doi.org/10.1177/0272431600020003004

Tracy, S. J. (2010). Qualitative quality: Eight 'big tent' criteria for excellent qualitative research. Qualitative Inquiry. 16, 837-851. DOI: https://doi.org/10.1177/1077800410383121

Villena, J. L. (2015). Reivindicando el presente desde la infancia. En Villena, J.L. \& Molina, E. (2015). Ciudades convida: Infancia, participacióny movilidad (pp.9-11). Barcelona: Graó.https://bit.ly/38kVvJL

Williams, D. R. (2016). Service-learning and the hungry and homeless: Tangible sensibilities of care among young urban adolescents. Children, Youth and Environments. 26(1), 164-178. DOI: http:// dx.doi.org/10.7721/chilyoutenvi.26.1.0164. 